\title{
The role of histological subtype in hormone receptor positive metastatic breast cancer: similar survival but different therapeutic approaches
}

\author{
Dorien Lobbezoo ${ }^{1,2}$, Wilfred Truin ${ }^{1,3}$, Adri Voogd ${ }^{1}$, Rudi Roumen ${ }^{1,3}$, Gerard \\ Vreugdenhil ${ }^{1,2}$, Marcus Wouter Dercksen ${ }^{2}$, Franchette van den Berkmortel ${ }^{4}$, Tineke \\ Smilde $^{5}$, Agnes van de Wouw ${ }^{6}$, Roel van Kampen ${ }^{1,7}$, Johanna van Riel $^{8}$, Natascha \\ Peters $^{9}$, Petronella Peer ${ }^{10}$, Vivianne C.G. Tjan-Heijnen ${ }^{1}$ \\ ${ }^{1}$ GROW, School for Oncology and Developmental Biology, Maastricht University Medical Center, Maastricht, The Netherlands \\ ${ }^{2}$ Department of Internal Medicine, Máxima Medical Center, Veldhoven, The Netherlands \\ ${ }^{3}$ Department of Surgery, Máxima Medical Center, Veldhoven, The Netherlands \\ ${ }^{4}$ Department of Internal Medicine, Atrium-Orbis, Heerlen, The Netherlands \\ ${ }^{5}$ Department of Internal Medicine, Jeroen Bosch Hospital, Den Bosch, The Netherlands \\ ${ }^{6}$ Department of Internal Medicine, VieCuri Medical Center, Venlo, The Netherlands \\ ${ }^{7}$ Department of Internal Medicine, Atrium-Orbis, Sittard, The Netherlands \\ ${ }^{8}$ Department of Internal Medicine, St Elisabeth Hospital, Tilburg, The Netherlands \\ ${ }^{9}$ Department of Internal Medicine, St Jans Hospital, Weert, The Netherlands \\ ${ }^{10}$ Department for Health Evidence, Radboud University Medical Center, Nijmegen, The Netherlands \\ Correspondence to: Vivianne C.G. Tjan-Heijnen, email: vcg.tjan.heijnen@mumc.nl
}

Keywords: metastatic breast cancer, histology, invasive lobular carcinoma, invasive ductal carcinoma, treatment

Received: December 06, 2015

Accepted: March 28, 2016

Published: April 19, 2016

ABSTRACT

Introduction: This study describes the differences between the two largest histological breast cancer subtypes (invasive ductal carcinoma (IDC) and invasive (mixed) lobular carcinoma (ILC) with respect to patient and tumor characteristics, treatment-choices and outcome in metastatic breast cancer.

Results: Patients with ILC were older at diagnosis of primary breast cancer and had more often initial bone metastasis $(46.5 \%$ versus $34.8 \%, P=0.01)$ and less often multiple metastatic sites compared to IDC $(23.7 \%$ versus $30.9 \%, P=0.11)$. Six months after diagnosis of metastatic breast cancer, $28.1 \%$ of patients with ILC and $39.8 \%$ of patients with IDC had received chemotherapy with a longer median time to first chemotherapy for those with ILC $(P=0.001)$. After six months $84.8 \%$ of patients with ILC had received endocrine therapy versus $72.5 \%$ of patients with IDC $(P=0.0001)$. Median overall survival was 29 months for ILC and 25 months for IDC $(P=0.53)$.

Materials and Methods: We included 437 patients with hormone receptorpositive IDC and 131 patients with hormone receptor-positive ILC, all diagnosed with metastatic breast cancer between 2007-2009, irrespective of date of the primary diagnosis. Patient and tumor characteristics and data on treatment and outcome were collected. Survival curves were obtained using the Kaplan-Meier method.

Conclusions: Treatment strategies of hormone receptor-positive metastatic breast cancer were remarkably different for patients with ILC and IDC. Further research is required to understand tumor behavior and treatment-choices in real-life. 


\section{INTRODUCTION}

The two most frequent histological subtypes of breast cancer are invasive ductal carcinoma (IDC) and invasive lobular carcinoma (ILC), with IDC comprising $75-80 \%$ and ILC $5-15 \%$ of all breast cancer cases. ILC is being associated with larger tumor size at presentation, more bilateral and multifocal involvement and with a different pattern of metastatic spread compared with IDC $[1,2]$. Furthermore, ILC is more often HR-positive, HER2negative, with lower S-phase fraction and less often positive for the tumor suppressor gene p53, compared with IDC [1]. Also, treatment response is known to be different. In a combined analysis on a number of retrospective series, the pathological complete response rate of neo-adjuvant chemotherapy was significantly lower in ILC $(1.7 \%)$ than in IDC (11.6\%) [3]. In the adjuvant setting, retrospective data suggest a higher efficacy of endocrine therapy for ILC than IDC [4]. More recently, a large retrospective study strongly suggested that HR-positive breast cancer patients with ILC do not seem to benefit from adjuvant chemotherapy in addition to endocrine therapy [5].

Whether these differences have a prognostic impact is controversial. Some studies found no effect of histology on survival $[1,6,7]$, others found a better prognosis for patients with ILC compared to those with IDC [8-10] and some found a change in prognosis over time with a better prognosis for ILC during the first years of follow-up and a worse prognosis during later years $[2,11]$.

So far, studies on histological subtypes consider early breast cancer and not metastatic breast cancer. The aim of this study was to describe the differences between IDC and ILC with respect to patient and tumor characteristics, treatment-choices and outcome in metastatic breast cancer. In order to account for the effect of the hormone receptor (HR) on outcome and treatment-decision making, we only included patients with HR-positive breast cancer.

\section{RESULTS}

\section{Patient characteristics}

Of the 568 patients with HR-positive metastatic breast cancer, $23 \%$ had (mixed type) ILC (131 patients), hereafter referred to as ILC and $77 \%$ had IDC (437 patients) (Table 1).

Metastatic breast cancer patients with ILC were older at the time of primary breast cancer diagnosis, compared with patients with IDC (median age at diagnosis 62 years for ILC versus 58 years for IDC, $P=0.03$ ). At initial presentation, patients with HR-positive ILC had larger tumors (T2-3, $62.5 \%$ versus $49.7 \%, P=0.002$ ) and slightly more node-positive disease (66\% versus $56.9 \%, P=0.10$ ), but with less often a HER2 positive status $(7.6 \%$ versus $15.3 \%, P=0.02$ ) and lower grade (not significant) compared with patients with HR-positive IDC. No differences were seen in use of adjuvant chemotherapy or endocrine therapy between the histological subtypes.

Bone was the most common initial site of distant metastasis in both HR-positive histological subtypes, although bone metastasis as initial site was more frequently observed for patients with ILC compared to those with IDC (46.5\% versus $34.8 \%, P=0.01)$. Fewer patients with ILC were diagnosed with multiple sites of distant metastasis compared to those with IDC $(23.7 \%$ versus $30.9 \%$, $P=0.11$ ).

\section{Palliative systemic treatment}

Median follow-up after diagnosis of metastatic disease was 37.1 months (range 5.2-54.6) with 239 patients $(42 \%)$ alive at the end of the follow-up period.

Time between diagnosis of HR-positive metastatic breast cancer and start of palliative chemotherapy was significantly longer for patients with ILC (median not yet reached) compared with IDC (median 16.9 months, 95\% Confidence Interval (CI) 9.6-22.3, $P=0.001$ ) (Figure 1A). Six months after diagnosis of HR-positive metastatic breast cancer less patients with ILC had received palliative chemotherapy compared with IDC (28.1\% versus $39.8 \%$ respectively).

Time between diagnosis of HR-positive metastatic breast cancer and start of palliative endocrine therapy was significantly shorter for patients with ILC (median, 0.6 months, $95 \%$ CI $0.5-0.8$ ) compared with patients with IDC (median, 1.1 months, 95\% CI 1.0-1.5, $P=0.0001$ ) (Figure 1B). Six months after diagnosis of metastatic breast cancer $84.8 \%$ of patients with ILC had received palliative endocrine therapy compared with $72.5 \%$ of patients with IDC.

\section{Outcome}

Median overall survival was 29.4 months $(95 \%$ CI 22.5-36.6) for patients with HR-positive ILC and 25.4 months $(95 \%$ CI $21.8-31.7)$ for patients with HR-positive IDC $(P=0.53)$.

In multivariable analysis for patients with ILC with palliative endocrine therapy and palliative chemotherapy as time-dependent covariates, early initiation of palliative chemotherapy was associated with an unfavorable survival (hazard ratio $2.8,95 \% \mathrm{CI} 1.7-4.6, P<.0001$ ) compared to no palliative chemotherapy during the observation period. Conversely, early initiation of palliative endocrine therapy was associated with a favorable survival (hazard ratio 0.4 , $95 \%$ CI $0.2-0.8, P=0.005)$ compared to no palliative endocrine therapy during the observation period.

In multivariable analysis for patients with IDC, early initiation of palliative chemotherapy was associated with an unfavorable survival (hazard ratio $2.1,95 \%$ CI 1.6-2.7. $P<.0001)$ when compared with no chemotherapy, whereas early treatment with palliative endocrine therapy was not 
Table 1: Baseline characteristics of HR+ metastatic breast cancer divided by histological subtype

\begin{tabular}{|c|c|c|c|c|c|}
\hline \multirow{2}{*}{ Characteristics } & \multicolumn{2}{|c|}{$\begin{array}{l}\text { Invasive ductal carcinoma } \\
\qquad N=437\end{array}$} & \multicolumn{2}{|c|}{$\begin{array}{c}\text { Invasive lobular and mixed } \\
\text { carcinoma } N=131\end{array}$} & \multirow[t]{2}{*}{$P$} \\
\hline & No & $\%$ & No & $\%$ & \\
\hline \multicolumn{6}{|l|}{ Age at primary diagnosis } \\
\hline Median (range) & \multicolumn{2}{|c|}{$58(25-91)$} & \multicolumn{2}{|c|}{$62(36-89)$} & 0.03 \\
\hline$<50$ years & 124 & 28.4 & 28 & 21.4 & 0.11 \\
\hline$\geq 50$ years & 313 & 71.6 & 103 & 78.6 & \\
\hline \multicolumn{6}{|c|}{ Histological grade of primary tumor } \\
\hline SBR 1 & 41 & 13.4 & 17 & 19.8 & 0.11 \\
\hline SBR 2 & 157 & 51.5 & 48 & 55.8 & \\
\hline SBR 3 & 107 & 35.1 & 21 & 24.4 & \\
\hline Unknown & 132 & & 45 & & \\
\hline \multicolumn{6}{|l|}{ Primary tumor stage } \\
\hline T1 & 157 & 48.2 & 29 & 30.2 & $<0.001$ \\
\hline $\mathrm{T} 2$ & 150 & 46.0 & 51 & 53.1 & \\
\hline $\mathrm{T} 3$ & 12 & 3.7 & 9 & 9.4 & \\
\hline $\mathrm{T} 4$ & 7 & 2.1 & 7 & 7.3 & \\
\hline Unknown & 111 & & 35 & & \\
\hline \multicolumn{6}{|l|}{ Regional lymph node stage } \\
\hline N0 & 141 & 43.1 & 34 & 34.0 & 0.005 \\
\hline N1 & 110 & 33.6 & 40 & 40.0 & \\
\hline $\mathrm{N} 2$ & 50 & 15.3 & 8 & 8.0 & \\
\hline N3 & 26 & 8.0 & 18 & 18.0 & \\
\hline Unknown & 110 & & 31 & & \\
\hline \multicolumn{6}{|c|}{ HER2 status of primary tumor } \\
\hline Positive & 67 & 15.3 & 10 & 7.6 & 0.02 \\
\hline Negative & 370 & 84.7 & 121 & 92.4 & \\
\hline \multicolumn{6}{|c|}{ Prior adjuvant endocrine therapy } \\
\hline Yes & 228 & 52.2 & 76 & 58.0 & 0.24 \\
\hline No & 209 & 47.8 & 55 & 42.0 & \\
\hline \multicolumn{6}{|l|}{ Prior adjuvant chemotherapy } \\
\hline Yes & 135 & 30.9 & 40 & 30.5 & 0.94 \\
\hline No & 302 & 69.1 & 91 & 69.5 & \\
\hline \multicolumn{6}{|c|}{ Prior adjuvant targeted therapy } \\
\hline Yes & 18 & 4.1 & 1 & 0.8 & 0.06 \\
\hline No & 419 & 95.9 & 130 & 99.2 & \\
\hline \multicolumn{6}{|c|}{ Metastatic-free interval (MFI) } \\
\hline Median (range), months & \multicolumn{2}{|c|}{$38.7(0-234)$} & \multicolumn{2}{|c|}{$32.3(0-321)$} & 0.58 \\
\hline De novo & 81 & 18.5 & 26 & 19.9 & 0.1 \\
\hline MFI $\leq 24$ months & 64 & 14.7 & 28 & 21.3 & \\
\hline MFI > 24 months & 292 & 66.8 & 77 & 58.8 & \\
\hline \multicolumn{6}{|l|}{ Initial site of metastasis } \\
\hline Bone & 152 & 34.8 & 61 & 46.5 & 0.01 \\
\hline Visceral & 114 & 26.1 & 30 & 22.9 & 0.50 \\
\hline Brain & 8 & 1.8 & 3 & 2.3 & 0.74 \\
\hline Skin and lymph nodes & 28 & 6.4 & 6 & 4.6 & 0.44 \\
\hline Multiple* & 135 & 30.9 & 31 & 23.7 & 0.11 \\
\hline
\end{tabular}

Abbreviations; HR+ hormone receptor positive, $N$ number, SBR Scarff Bloom Richardson, MFI metastatic-free interval

$* \geq 1$ of the aforementioned initial sites of metastasis. 
associated with survival (hazard ratio $0.9,95 \%$ CI 0.6-1.2, $P=0.4)$.

\section{Residual survival: six months after diagnosis}

For ILC, the residual survival was significantly longer for patients not treated with palliative chemotherapy within the first six months after diagnosis of metastatic breast cancer (median 44.0 months, 95\% CI 30.2-not yet reached) versus patients treated with palliative chemotherapy (median 15.2 months, 95\% CI 7.8-19.2) (Figure 2A). For IDC, residual survival when not treated with palliative chemotherapy was 41.8 (95\% CI 33.3-not yet reached) compared with 16.8 months (95\% CI 13.6-22.5) for patients treated with palliative chemotherapy within the first six months after diagnosis of metastatic breast cancer (Figure 2B).

Reversely, for ILC-patients with palliative endocrine treatment within the first six months after diagnosis of metastatic breast cancer residual survival was 37.5 months (95\% CI 26.9-not yet reached) compared with 11.2 months (95\% CI 3.0-20.4) for patients with HR-positive metastatic ILC without palliative endocrine therapy before the first six months (Figure 3A). For patients with HRpositive metastatic IDC treated with palliative endocrine therapy during the first six months, residual survival was 33.5 months (95\% CI 25.7-not yet reached) compared with 18.9 months (95\% CI 14.0-26.8) for patients with HRpositive metastatic IDC without palliative endocrine therapy in the first six months of metastatic disease (Figure 3B).

\section{DISCUSSION}

To our knowledge, the role of histological subtype in palliative systemic treatment of metastatic breast cancer has not been studied before. In a cohort of 568 HR-positive metastatic breast cancer patients, we showed that patients with HR-positive ILC had more often bone and less often multiple sites as initial site of distant metastasis compared to those with HR-positive IDC. Six months after metastatic diagnosis, patients with HR-positive ILC had received significantly less often chemotherapy (28\% versus $40 \%)$ and more often endocrine therapy ( $85 \%$ versus $73 \%)$ as compared to patients with HR-positive IDC. Patients starting with palliative chemotherapy during the first six months had a significantly shorter median residual survival thereafter as compared to those who did not (15 months versus 44 months for ILC, and 17 and 42 months for IDC, respectively). Reversely, patients treated with endocrine therapy in the first six months had a longer median residual survival compared to those who did not (37 months versus 11 months for ILC and 33 and 19 months for IDC, respectively). Our results confirm that, in addition to HRstatus, histology correlates with presentation of metastatic disease by number and type of distant metastatic sites, and thereby affects treatment-decision making in real-life.

The intriguing scientific question is to determine how histological subtype influences the metastatic spread in HR-positive breast cancer. It is hypothesized that the loss of E-cadherin, a cell-cell adhesion molecule, in ILC may result in less adhesiveness of the tumor cells and therefore disseminate and infiltrate certain distant locations more easily [1].

In this HR-positive metastatic breast cancer cohort we also looked at the association of palliative systemic treatment with survival of patients with metastatic breast cancer for both histological subtypes. With the favorable presentation of distant metastasis by number and location of patients with HR-positive ILC as compared to HR-positive IDC, one would expect a more indolent disease course and a better outcome. However, we showed that for patients with HR-positive breast cancer, overall disease outcome was comparable irrespective of histology and irrespective of different treatment choices made in real-life, as discussed above. And interestingly, the efficacy of chemotherapy - once the treatment choice was made - was comparable irrespective of histology. However, treatment with palliative endocrine therapy was only associated with a favorable prognosis of
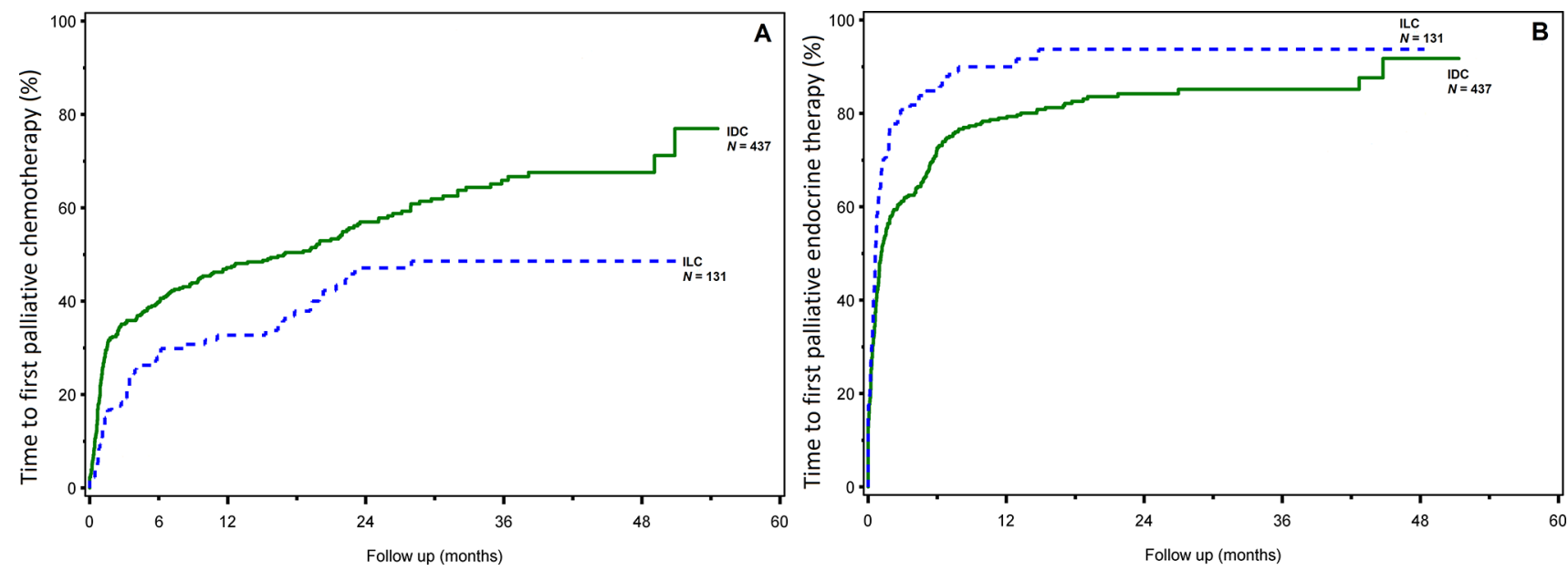

Figure 1: Time to first palliative chemotherapy (A) and endocrine therapy (B) by histological subtype. 
patients with HR-positive ILC, whereas this was not seen in patients with HR-positive IDC, also suggesting the impact of histology over HR-status. This is in concordance with the early breast cancer setting, in which there is evidence suggesting differences in efficacy of systemic therapy between the two histological subtypes [3-5, 12].

Although there is some proof that histology could be helpful in treatment decision-making [13-15], the current guidelines do not include histological subtype as an indicator for the use of systemic treatment in general, or for a specific regimen $[16,17]$. Interestingly, in the adjuvant setting, histology has been shown to be of importance when deciding between the use of breast-conserving surgery or mastectomy after neo-adjuvant chemotherapy [18]. The current study shows that histological subtype can be of predictive value for HR-positive metastatic breast cancer with regards to the effectiveness of early initiation of palliative endocrine therapy. It may be possible that in patients with HR-positive lobular breast cancer, the higher incidence of bone metastases as initial metastatic site partly accounts for the initial choice of endocrine therapy. It underlines the relevance of acknowledging a different metastatic pattern, and thereby different initial treatment choices, between breast cancer patients with ductal versus lobular histology.

Much more than in the adjuvant setting, treatment decisions in the metastatic setting are based on the observed and anticipated clinical course of the disease, which is not only determined by the tumor characteristics as described earlier. Also age, performance status, previous therapies and toxicities, comorbidity and patient and doctor preferences play a role. The complexity of this process, together with the retrospective design of our study make it impossible to identify and rule out confounding by indication.
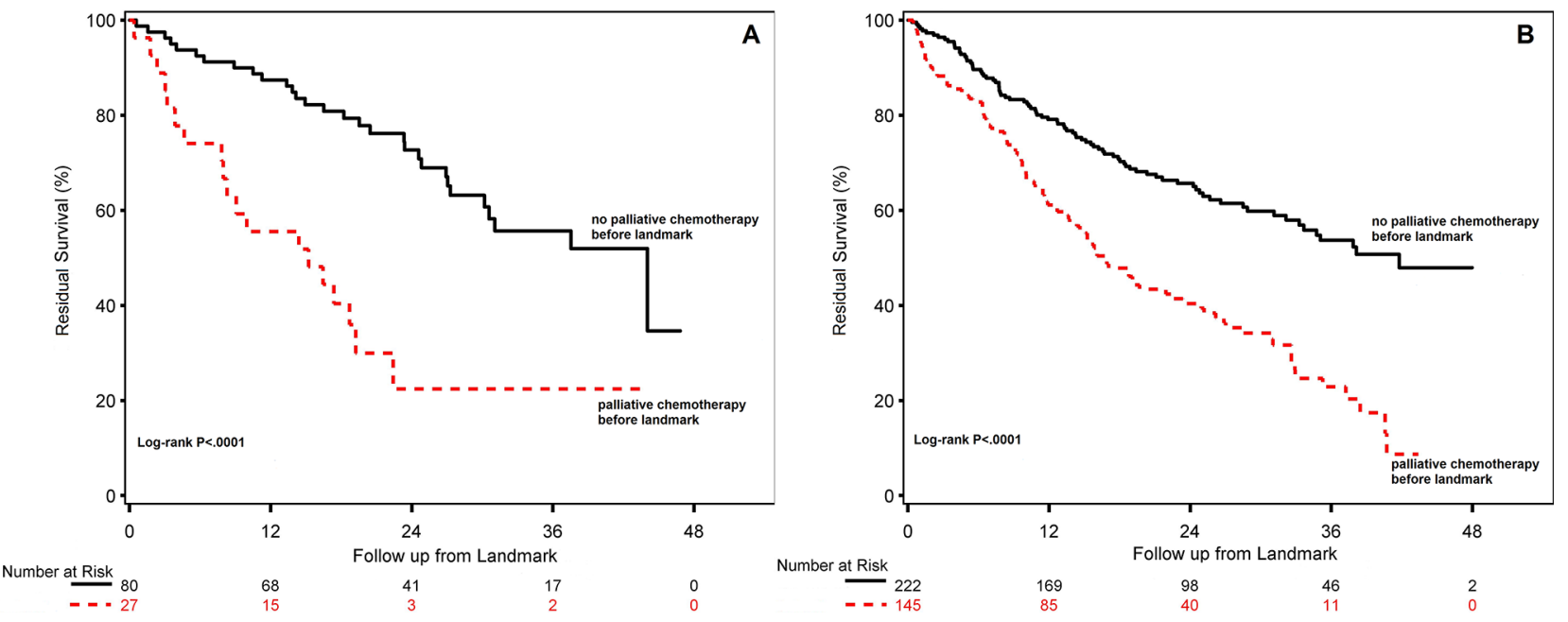

Figure 2: Residual survival for patients with HR-positive ILC (A) and HR-positive IDC (B) treated with or without any palliative chemotherapy during the first six months after diagnosis of metastatic breast cancer.
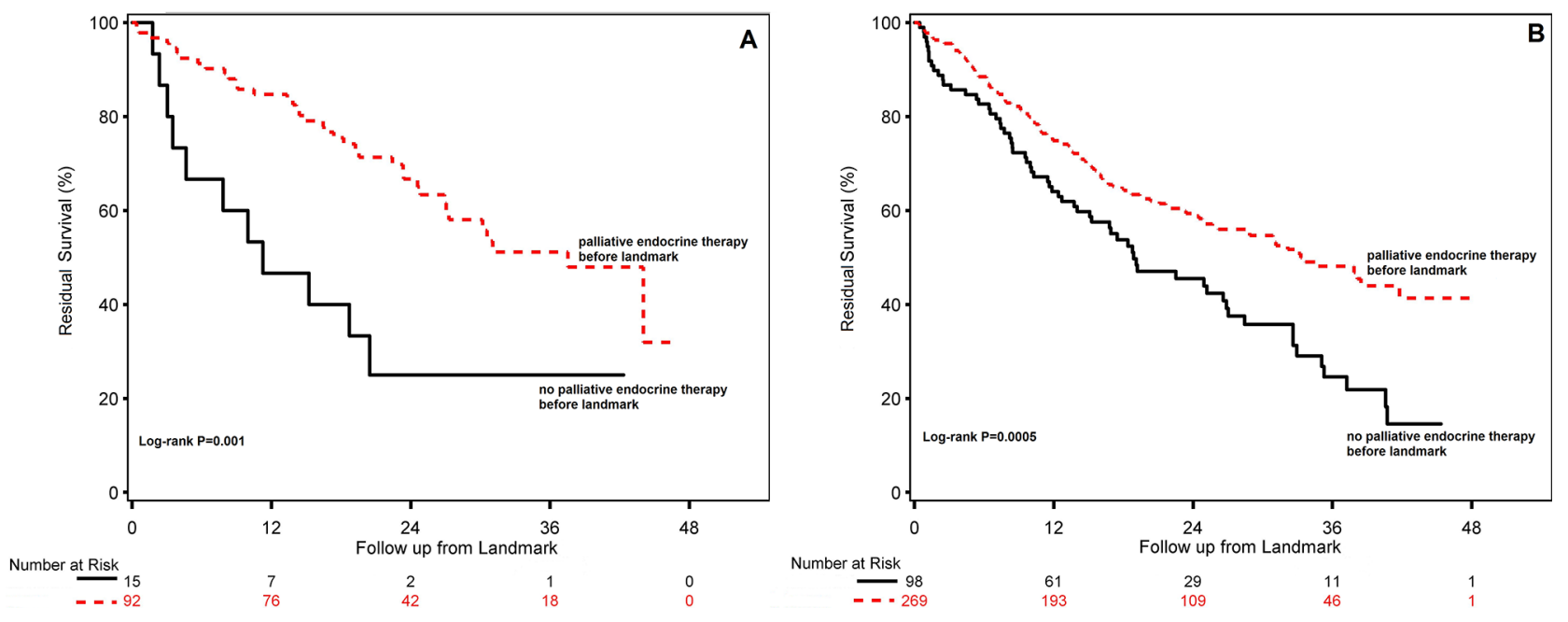

Figure 3: Residual survival for patients with HR-positive ILC (A) and HR-positive IDC (B) treated with or without any palliative endocrine therapy during the first six months after diagnosis of metastatic breast cancer. 
Furthermore, localization of the metastases (visceral versus bone metastasis for instance) could be related to symptomatology and thereby timing of detection, and could therefore have introduced lead time bias.

In this study patients with pure lobular carcinoma and mixed lobular carcinomas were combined for the analyses. In other studies on histological subtypes of breast cancer mixed and lobular carcinoma had similar outcome $[14,19]$. Even with combining these subgroups, the proportion of HER2-positive tumors was too low to further analyze anti-HER2 therapy. In the adjuvant treatment setting the magnitude of benefit from adjuvant trastuzumab between patients with ILC and IDC was shown not to be different [20]. Another limitation of our study is that patient numbers were too small to perform analysis on specific chemotherapy regimens. Microarray analysis have demonstrated that ILC and IDC can be distinguished on the basis of genomic and expression profiles [21]. The increasing knowledge on genomic differences between ILC and IDC can help to answer questions on in vivo chemosensitivity. For example, topoisomerase-II $\alpha$ gene amplification is a predictive biomarker for response to anthracyclines and in ILC this gene amplification is lacking, which could help understand the poor responsiveness of ILC to neo-adjuvant chemotherapy, including anthracyclines [22]. This genetic information can help guide further research and may eventually be useful in making treatment decisions for histological breast cancer subtypes.

In conclusion, to our knowledge, this is the first study that investigates the role of histological subtype in HR-positive metastatic breast cancer. Although survival was comparable for the two histological subtypes, this was achieved with different treatment strategies. As patients with HR-positive ILC were less likely to receive chemotherapy than those with HR-positive IDC, histology may be a relevant factor in treatment-decision making. For a more definite conclusion on the role of histology, we recommend to incorporate histological subtype as a stratification factor in future clinical trials.

\section{MATERIALS AND METHODS}

\section{Patient selection}

We identified all patients diagnosed with metastatic breast cancer between 2007-2009 in eight hospitals in the South-East of the Netherlands. All patients with metastatic breast cancer were selected irrespective of the date of primary breast cancer diagnosis (also including patients with de novo metastatic breast cancer; $18.5 \%$ of patients with IDC and $19.9 \%$ of patients with ILC), with the exception of patients with a diagnosis of primary breast cancer before 1990, due to limitations in the availability of data on the primary tumor and initial treatment.

Histology was classified according to the International Classification of Diseases for Oncology
(ICD-O) [23]. ILC and mixed histology were defined as code 8520 and 8522 . IDC was defined as code 8500 . From the total of 815 metastatic breast cancer patients, we excluded 76 patients with either unknown histology or histological subtypes other than IDC or ILC. Of the remaining 739 metastatic breast cancer patients, we excluded 171 patients with HR-negative tumors (27\% of patients with IDC and $8 \%$ of patients with ILC) in order to rule out the impact of HR status. In total, our study population consisted of 568 patients divided in two groups based on histology; one group of 437 patients with IDC and the other group of 131 patients with ILC.

\section{Data collection}

Information was collected on patient and tumor characteristics, treatment and outcome. Tumors were characterized by the sixth edition of the TNM classification of malignant tumors [24] and Scarff Bloom Richardson (SBR) histological grading [25]. Estrogen receptor (ER) and progesterone receptor (PR) positivity was defined as positive nuclear staining of $\geq 10 \%$. HER2 positivity was defined as immunohistochemistry score of $3+$ or $2+$ with positive FISH. In case of missing HER2 status a dedicated pathologist centrally reviewed missing data when material was available. Initial sites of metastasis were categorized as: bone, visceral (including lung, liver, pleural, peritoneal, pericardial and lymphangitic carcinomatosis), brain (including leptomeningeal and CNS), skin and lymph nodes, and multiple metastases (more than one of the metastatic sites).

\section{Statistical analysis}

Baseline characteristics between the two histological groups were compared using chi-square tests for categorical variables and Wilcoxon rank sum tests for continuous variables.

Metastatic-free interval was defined as time between date of primary diagnosis and date of first distant metastasis. Overall survival after diagnosis of metastatic breast cancer was defined as time between date of first distant metastasis and date of death. Survival curves and time to first palliative systemic therapy (either chemotherapy or endocrine therapy) were estimated using the Kaplan-Meier method and compared using log-rank tests. All patients still alive were censored at the date of last follow-up of each individual patient. Patients who died without palliative therapy were censored at the date of death in the analysis of time to first palliative therapy.

To explore the association of palliative systemic therapy with the survival of patients with metastatic breast cancer for both histological subtypes a Cox proportional hazards model was performed with palliative chemotherapy and endocrine therapy as a time-dependent covariate, since the administration of treatment can change over time and is dependent on the time available for each patient to 
receive the treatment. We did not explore the association between palliative targeted therapy, such as trastuzumab and bevacizumab, and survival since the number of patients with IDC and ILC receiving targeted therapy was very low. Since all these patients received targeted therapy with chemotherapy, these patients were included in the analysis on initial chemotherapy. In addition, the landmark method was used to estimate survival after a specific time-point, the so-called residual survival [26]. As we were interested to learn about the obtained survival in relation to the initial palliative treatment choices, we chose six months after diagnosis of metastatic breast cancer as landmark. Consequently, patients who already died within 6 months were excluded for the residual survival curves.

All analyses were performed using SAS version 9.2. All reported $P$-values are two-sided and $P$-value $\leq 0.05$ was considered statistically significant.

\section{ACKNOWLEDGMENTS}

We thank Wim A.J.G. Lemmens for his assistance with performing the statistical analyses.

\section{FUNDING}

This work was supported by the Netherlands Organization for Health Research and Development (ZonMw: 80-82500-98-8003) and the Department Medical Oncology Maastricht University Medical Centre, The Netherlands

\section{CONFLICTS OF INTEREST}

The authors have declared no conflicts of interest.

\section{REFERENCES}

1. Arpino G, Bardou VJ, Clark GM, Elledge RM. Infiltrating lobular carcinoma of the breast: tumor characteristics and clinical outcome. Breast cancer res. 2004; 6:R149-56.

2. Pestalozzi BC, Zahrieh D, Mallon E, Gusterson BA, Price KN, Gelber RD, Holmberg SB, Lindtner J, Snyder R, Thurlimann B, Murray E, Viale G, Castiglione-Gertsch M, et al. Distinct clinical and prognostic features of infiltrating lobular carcinoma of the breast: combined results of 15 International Breast Cancer Study Group clinical trials. J Clin Oncol 2008; 26: 3006-3014.

3. Katz A, Saad ED, Porter P, Pusztai L. Primary systemic chemotherapy of invasive lobular carcinoma of the breast. Lancet Oncol. 2007; 8:55-62.

4. Rakha EA, El-Sayed ME, Powe DG, Green AR, Habashy H, Grainge MJ, Robertson JF, Blamey R, Gee J, Nicholson RI, Lee AH, Ellis IO. Invasive lobular carcinoma of the breast: response to hormonal therapy and outcomes. Eur J Cancer. 2008; 44:73-83.
5. Truin W, Voogd AC, Vreugdenhil G, van der Heiden-van der Loo,M., Siesling S, Roumen RM. Effect of adjuvant chemotherapy in postmenopausal patients with invasive ductal versus lobular breast cancer. Ann Oncol. 2012; 23: 2859-2865.

6. Fortunato L, Mascaro A, Poccia I, Andrich R, Amini M, Costarelli L, Cortese G, Farina M, Vitelli C. Lobular breast cancer: same survival and local control compared with ductal cancer, but should both be treated the same way? analysis of an institutional database over a 10 -year period. Ann Surg Oncol. 2012; 19:1107-1114.

7. Kwast AB, Groothuis-Oudshoorn KC, Grandjean I, Ho VK, Voogd AC, Menke-Pluymers MB, van der Sangen MJ, TjanHeijnen VC, Kiemeney LA, Siesling S. Histological type is not an independent prognostic factor for the risk pattern of breast cancer recurrences. Breast Cancer Res Treat. 2012; 135:271-280.

8. Cristofanilli M, Gonzalez-Angulo A, Sneige N, Kau SW, Broglio K, Theriault RL, Valero V, Buzdar AU, Kuerer H, Buccholz TA, Hortobagyi GN. Invasive lobular carcinoma classic type: response to primary chemotherapy and survival outcomes. J Clin Oncol. 2005; 23:41-48.

9. Dian D, Herold H, Mylonas I, Scholz C, Janni W, Sommer H, Friese K. Survival analysis between patients with invasive ductal and invasive lobular breast cancer. Arch Gynecol Obstet. 2009; 279:23-28.

10. Wasif N, Maggard MA, Ko CY, Giuliano AE. Invasive lobular vs. ductal breast cancer: a stage-matched comparison of outcomes. Ann Surg Oncol. 2010; 17:1862-1869.

11. Rakha EA, El-Sayed ME, Menon S, Green AR, Lee AH, Ellis IO. Histologic grading is an independent prognostic factor in invasive lobular carcinoma of the breast. Breast Cancer Res Treat. 2008; 111:121-127.

12. Farese SA, Aebi S. Infiltrating lobular carcinoma of the breast: systemic treatment. Breast dis. 2008; 30:45-52.

13. Purushotham A, Pinder S, Cariati M, Harries M, Goldhirsch A. Neoadjuvant chemotherapy: not the best option in estrogen receptor-positive, HER2-negative, invasive classical lobular carcinoma of the breast?. J Clin Oncol. 2010; 28:3552-3554.

14. Colleoni M, Rotmensz N, Maisonneuve P, Mastropasqua MG, Luini A, Veronesi P, Intra M, Montagna E, Cancello G, Cardillo A, Mazza M, Perri G, Iorfida M, et al. Outcome of special types of luminal breast cancer. Ann Oncol. 2012; 23:1428-1436.

15. Akiyama F, Horii R. Therapeutic strategies for breast cancer based on histological type. Breast cancer. 2009; 16:168-172.

16. Cardoso F, Costa A, Norton L, Senkus E, Aapro M, Andre F, Barrios CH, Bergh J, Biganzoli L, Blackwell KL, Cardoso MJ, Cufer T, El Saghir N, et al. ESO-ESMO 2nd international consensus guidelines for advanced breast cancer (ABC2). Breast. 2014; 23:489-502.

17. Gradishar WJ, Anderson BO, Blair SL, Burstein HJ, Cyr A, Elias AD, Farrar WB, Forero A, Giordano SH, Goldstein LJ, 
Hayes DF, Hudis CA, Isakoff SJ, et al. National Comprehensive Cancer Network Breast Cancer Panel,. Breast cancer version 3.2014. Journal of the National Comprehensive Cancer Network. 2014; 12:542-90.

18. Truin W, Vugts G, Roumen RM, Maaskant-Braat AJ, Nieuwenhuijzen GA, van der Heiden-van der Loo,M., TjanHeijnen VC, Voogd AC. Differences in Response and Surgical Management with Neoadjuvant Chemotherapy in Invasive Lobular Versus Ductal Breast Cancer. Ann Surg Oncol. 2015.

19. Bharat A, Gao F, Margenthaler JA. Tumor characteristics and patient outcomes are similar between invasive lobular and mixed invasive ductal/lobular breast cancers but differ from pure invasive ductal breast cancers. Am J Surg. 2009; 198:516-519.

20. Metzger-Filho O, Procter M, de Azambuja E, Leyland-Jones B, Gelber RD, Dowsett M, Loi S, Saini KS, Cameron D, Untch M, Smith I, Gianni L, Baselga J, et al. Magnitude of trastuzumab benefit in patients with HER2-positive, invasive lobular breast carcinoma: results from the HERA trial. J Clin Oncol. 2013; 31:1954-1960.

21. Bertucci F, Orsetti B, Negre V, Finetti P, Rouge C, Ahomadegbe JC, Bibeau F, Mathieu MC, Treilleux I, Jacquemier J, Ursule L, Martinec A, Wang Q, et al. Lobular and ductal carcinomas of the breast have distinct genomic and expression profiles. Oncogene. 2008; 27:5359-5372.
22. Brunello E, Brunelli M, Manfrin E, Nottegar A, Bersani S, Vergine M, Molino A, Fiorio E, Chilosi M, Gobbo S, Martignoni G, Bonetti F. Classical lobular breast carcinoma consistently lacks topoisomerase-IIalpha gene amplification: implications for the tailored use of anthracycline-based chemotherapies. Histopathology. 2012; 60:482-488.

23. World Health Organization. International statistical classification of diseases and related health problems (ICD10), 10th revision. Geneva. 2010.

24. Greene FL, Page DL, Fleming ID, Fritz A, Balch CM, Haller DG, Morrow M. AJCC cancer staging manual. Springer New York. 2002.

25. Elston CW, Ellis IO. Pathological prognostic factors in breast cancer. I. The value of histological grade in breast cancer: experience from a large study with long-term follow-up. Histopathology. 1991; 19:403-410.

26. Anderson JR, Cain KC, Gelber RD. Analysis of survival by tumor response. J Clin Oncol. 1983; 1:710-719. 